\title{
Determination of the muonic branching ratio of the $W$ boson and its total width via cross-section measurements at the Tevatron and LHC
}

\author{
Stefano Camarda ${ }^{1, a}$, Jakub Cuth ${ }^{2}$, Matthias Schott ${ }^{2}$ \\ ${ }^{1}$ CERN, Geneva, Switzerland \\ 2 Johannes Gutenberg University, Mainz, Germany
}

Received: 25 August 2016 / Accepted: 24 October 2016 / Published online: 9 November 2016

(c) The Author(s) 2016. This article is published with open access at Springerlink.com

\begin{abstract}
The total $W$-boson decay width $\Gamma_{W}$ is an important observable which allows testing of the standard model. The current world average value is based on direct measurements of final state kinematic properties of $W$-boson decays, and has a relative uncertainty of $2 \%$. The indirect determination of $\Gamma_{W}$ via the cross-section measurements of vectorboson production can lead to a similar accuracy. The same methodology leads also to a determination of the leptonic branching ratio. This approach has been successfully pursued by the CDF and D0 experiments at the Tevatron collider, as well as by the CMS collaboration at the LHC. In this paper we present for the first time a combination of the available measurements at hadron colliders, accounting for the correlations of the associated systematic uncertainties. Our combination leads to values of $\mathrm{BR}(W \rightarrow \mu \nu)=(10.72 \pm 0.16) \%$ and $\Gamma_{W}=2113 \pm 31 \mathrm{MeV}$, respectively, both compatible with the current world averages.
\end{abstract}

\section{Introduction}

Precise measurements of the $W$-boson properties, such as its mass $m_{W}$ and its decay width $\Gamma_{W}$, allows testing of the standard model of particle physics. As a matter of fact, the relation between the $W$-boson mass, $m_{W}$, the top-quark mass, $m_{t}$, and the Higgs-boson mass, $m_{H}$, via loop corrections, allowed a prediction of the mass of the Higgs boson with an uncertainty smaller than $25 \mathrm{GeV}$. Models beyond the standard model could alter the relation between $m_{W}$ and $m_{H}$, since new particles can appear in virtual loops. Similarly, the total decay width of the $W$ boson can be altered by new particles.

Within the standard model, the total decay width of the $W$ boson is predicted to be equal to the sum of the partial widths

\footnotetext{
a e-mail: stefano.camarda@cern.ch
}

over three generations of lepton doublets and two generations of quark doublets. The partial widths are expressed as

$\Gamma_{W \rightarrow f \bar{f}^{\prime}}=\frac{\left|M_{f \bar{f}^{\prime}}\right|^{2} \cdot N_{C} \cdot G_{F} \cdot m_{W}^{3}}{6 \pi \sqrt{2}}\left(1+\delta_{f}^{\mathrm{rad}}\right)$

where $M_{f \bar{f}^{\prime}}=1, N_{C}=1$ for leptonic decays, $M_{f \bar{f}^{\prime}}$ corresponds to the CKM matrix elements, and $N_{C}=3$. $\left(1+\alpha_{S}\left(m_{W}\right) / \pi+\cdots\right)$ is the colour factor for the quarkdecay modes [1]. Radiative corrections are represented by $\delta_{f}^{\text {rad }}$. They depend, among other parameters, on the topquark mass, $m_{t}$, and the Higgs-boson mass, $m_{H}$, and are small in the standard model (SM) since a large part of the corrections is absorbed in the measured values of $G_{F}=$ $1.1663787(8) \cdot 10^{-5} \mathrm{GeV}^{-2}$ and $m_{W}=80.385 \pm 0.015 \mathrm{GeV}$. The radiative corrections correspond to $\delta_{\ell}^{\text {rad }} \approx 0.34 \%$ for leptons and of $\delta_{q}^{\mathrm{rad}} \approx 0.40 \%$ for quarks [2]. New particle candidates that couple to the $W$ boson and are lighter than $m_{W}$, would open a new decay channel and alter $\Gamma_{W}$. One very prominent example is supersymmetric models in which the $W$ boson can decay to the lightest super-partner of the charged gauge bosons and the lightest super-partner of the neutral gauge bosons. Hence a precise measurement of $\Gamma_{W}$ might reveal physics beyond the standard model. In addition, assuming standard model relations, the dependence of the partial and total widths of the $W$ boson on the strongcoupling constant allows to determine the value of $\alpha_{s}$ from the hadronic and leptonic branching ratios of the $W$ boson [3].

The total width of the $W$ boson can be measured directly by kinematic fits to the measured decay lepton spectra, such as the transverse momentum of the charged lepton decay $p_{T}$ or the high-mass tail of the transverse mass $m_{T}$ as was performed at CDF and D0 [4-6], or via fits to the invariant mass distributions in the $q q q q$ and $q q l v$ final states as was done at the LEP experiments [7-11]. A combination of these 
direct results, based on kinematic measurements, leads to $\Gamma_{W}=2085 \pm 42 \mathrm{MeV}$, which is currently used as world average value [12].

An independent determination of the width of the $W$ boson is based on the measurement of the ratio of cross sections of $W$ - and $Z$-boson production in hadron collisions, defined as

$R=\frac{\sigma\left(p p^{\prime} \rightarrow W+X\right) \cdot \mathrm{BR}(W \rightarrow \ell v)}{\sigma\left(p p^{\prime} \rightarrow Z+X\right) \cdot \mathrm{BR}(Z \rightarrow \ell \ell)}$,

where $\operatorname{BR}\left(V \rightarrow \ell \ell^{\prime}\right)=\Gamma_{V \rightarrow \ell \ell^{\prime}} / \Gamma_{V}$ denotes the leptonic branching ratio of the vector-boson $(V=W, Z)$ decays. The ratio $R$ can be written as

$R=\frac{\sigma_{W}}{\sigma_{Z}} \cdot \frac{\Gamma_{W \rightarrow \ell v}}{\Gamma_{W}} \cdot \frac{\Gamma_{Z}}{\Gamma_{Z \rightarrow \ell \ell}}$,

where the total cross-section ratio $\sigma_{W} / \sigma_{Z}$ is known theoretically to high accuracy [13]. The ratio $\sigma_{Z \rightarrow \ell \ell} / \sigma_{Z}$ was precisely measured by the LEP experiments and therefore the leptonic branching ratio of the $W$ boson, $\mathrm{BR}(W \rightarrow \ell v)=$ $\Gamma_{W \rightarrow \ell v} / \Gamma_{W}$, can be inferred from the measurement of $R$. The advantage of extracting $\mathrm{BR}(W \rightarrow \ell v)$ from the crosssection ratio $R$ lies in the fact that many experimental systematic uncertainties of each vector-boson cross-section measurement, such as the uncertainty on the integrated luminosity, are highly correlated and cancel in the ratio. The leptonic width of the $W$ boson in the SM can be predicted by Eq. (1) and is $\Gamma(W \rightarrow \ell v)=226.5 \pm 0.1 \mathrm{MeV} .{ }^{1}$ The dominant uncertainty is due to the accuracy of $m_{W}$. Using this value, the total width of the $W$ boson can be extracted by a measurement of the leptonic branching ratio. This approach for the determination of the $W$-boson width was already pursued by the CDF [14], D0 [15], and CMS [16,17] experiments, leading to measurements of $\Gamma_{W}$ which have an accuracy comparable to the current world average.

In this paper we present a procedure for a first combination of the individual measurements of the muonic branching ratio of the $W$ boson and of $\Gamma_{W}$, accounting for the correlations of the individual systematic uncertainties. We have chosen to focus on the muon decay channel, as it has smaller experimental uncertainties.

The paper is structured as follows: we introduce the basic methodology in Sect. 2 and discuss the selected measurements for the combination in Sect. 3, where we also derive the corresponding fiducial cross-section ratios. The theoretical predictions of the cross-section ratios are discussed in Sect. 4 and the final extraction and combination of $\Gamma_{W}$ for the different experiments is presented in Sect. 5. The paper concludes with a brief summary and a discussion of the consistency of the results with the direct measurements and with the global electroweak fit in Sect. 6.

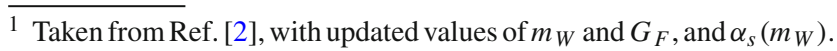

\section{Methodology}

The production cross section of $W$ and $Z$ bosons in hadron collisions is described by the Drell-Yan process [18] and can be experimentally defined as

$$
\begin{aligned}
\sigma_{\text {incl }}\left(p p \rightarrow V+X \rightarrow \ell \ell^{\prime}\right) & =\frac{N_{\text {Cand }}-N_{\mathrm{Bkg}}}{\epsilon \cdot \int L d t} \\
& =\frac{N_{\text {Cand }}-N_{\mathrm{Bkg}}}{C \cdot A \cdot \int L d t},
\end{aligned}
$$

where $N_{\mathrm{Cand}}$ and $N_{\mathrm{Bkg}}$ are the number of vector-boson candidates and the expected background events, respectively, and $\int L d t$ is the integrated luminosity of the corresponding data sample. The factor $\epsilon$ is the efficiency of the signal events passing the signal selection criteria, which is typically estimated with simulated samples of the signal process, and corrected for differences in the detector response between data and MC simulation. The efficiency correction $\epsilon$ can be decomposed as the product of a fiducial acceptance, $A$, and a detectorinduced correction factor, $C$, i.e. $\epsilon=A \cdot C$. The fiducial acceptance is the ratio of the number of events that pass the geometrical and kinematic requirements in the analysis at generator level over the total number of generated events in a simulated sample of signal process. The advantage of this decomposition is the separation to a large extent of detector and analysis related uncertainties, which enter the factor $C$, while all model and theoretical uncertainties, such as QCD scales and parton density function (PDF) uncertainties, enter $A$. The fiducial production cross section $\sigma_{\text {fid }}$ within the detector acceptance volume defined by A, is barely affected by model uncertainties, and is related to the fully inclusive cross section by $\sigma_{\text {fid }}=\sigma_{\text {incl }} \cdot A$.

The strategy for the combination of several indirect $\mathrm{BR}(W \rightarrow \mu \nu)$ and $\Gamma_{W}$ measurements from various experiments is therefore based on the measured fiducial crosssection ratio

$R_{\mathrm{fid}}=\frac{\sigma_{\mathrm{fid}}\left(p p^{\prime} \rightarrow W+X\right) \cdot \mathrm{BR}(W \rightarrow \ell v)}{\sigma_{\mathrm{fid}}\left(p p^{\prime} \rightarrow Z+X\right) \cdot \mathrm{BR}(Z \rightarrow \ell \ell)}$,

which has only negligible model uncertainties and uncorrelated experimental uncertainties between the different experiments. The fiducial ratio $R_{\mathrm{fid}}$ can be related to the inclusive ratio $R$, by

$R=\left(\frac{A_{W}}{A_{Z}}\right)^{-1} \cdot R_{\mathrm{fid}}$,

where the $A_{W}$ and $A_{Z}$ are the acceptance correction factors for the $Z$ - and $W$-boson analyses, respectively. Some published results only present a value for the inclusive crosssection ratio $R$, but do not publish a value for $R_{\text {fid }}$. In these cases, we have used the fiducial volume definition, the PDF set, and the $\mathrm{MC}$ generator of the corresponding analysis that were used to extract the acceptance ratio $A_{W} / A_{Z}$, in order to 
reconstruct the value of $R_{\mathrm{fid}}$. The uncertainty on the extrapolated values of $R_{\text {fid }}$ is estimated by subtracting the published model and PDF uncertainties from the total uncertainty on $R$.

Once the fiducial ratios are determined for each measurement, we can coherently predict the acceptance correction ratios $A_{W} / A_{Z}$ and the inclusive fiducial cross-section ratios $\sigma_{W} / \sigma_{Z}$, and extract the corresponding branching ratio $\mathrm{BR}(W \rightarrow \mu \nu)$ and decay width $\Gamma_{W}$ from the measurements of $R_{\mathrm{fid}}$. Each model variation, e.g. one particular eigenvector variation of a given PDF set, leads to new predictions of $A_{W} / A_{Z}$ and $\sigma_{W} / \sigma_{Z}$, thus also to new determined values of $\mathrm{BR}(W \rightarrow \mu \nu)$ and $\Gamma_{W}$ for each experiment. The measurements are combined treating the experimental uncertainties as uncorrelated, and the PDF and model uncertainties with a correlation model based on a common baseline for the theoretical predictions.

\section{Measurements used for the combination}

One of the first precise measurements of the $\sigma_{W} / \sigma_{Z}$ crosssection ratio was published by the $\mathrm{D} 0$ collaboration in proton anti-proton collisions at a centre-of-mass energy of $\sqrt{s}=$ $1.8 \mathrm{TeV}$ [15]. However, this measurement was performed only in the electron decay channel and hence is not used for this combination. The most precise measurement at the Tevatron collider was performed by the CDF collaboration at $\sqrt{s}=1.96 \mathrm{TeV}$ [14], using the electron and muon decay channels. Only the inclusive cross-section ratio was published (Table 1), but the clear definition of the fiducial volume, as reported in the paper, allows the extrapolation of the value of $R_{\text {fid }}$. The extrapolation factor $A_{W} / A_{Z}$ is estimated using the PYTHIA 6.2 [19] generator with the CTEQ5L PDF set [20].

Several measurements of $R$ were performed at the LHC by the CMS and ATLAS collaborations in proton-proton collisions at $\sqrt{s}=7 \mathrm{TeV}$ and $\sqrt{s}=8 \mathrm{TeV}[16,17,21]$, which are all used for the combination. In contrast to the Tevatron experiments, fiducial ratios together with a fiducial volume definition have also been published by the ATLAS and CMS experiments, as summarised in Table 1 . Hence no additional extrapolation to $R_{\mathrm{fid}}$ is performed for these measurements.

\section{Theoretical predictions and systematic uncertainties}

The total $W$ - and $Z$-boson production cross sections and their ratio, corresponding to the fiducial volume definitions of Table 1, are calculated at next-to-next-to-leading order in the perturbative expansion of the strong-coupling constant with FEWZ [22] using the MMHT2014 PDF set [23]. The calculations are based on the $G_{\mu}$ electroweak parameter scheme and the strong-coupling constant at the $Z$-boson mass is set to $\alpha_{s}\left(m_{Z}\right)=0.118$, as used in the MMHT2014 PDF determination. ${ }^{2}$ The uncertainties of the PDF set are estimated by a reevaluation of the predicted cross-section ratio for each error eigenvector within the MMHT2014 PDF set, as well as the comparison to the central prediction using a second PDF set, which is chosen to be the CT10 [24] in this study. The uncertainties, at $68 \% \mathrm{CL}$, include contributions from the strong-coupling constant $\alpha_{S}$ as well as variations of the renormalisation scale, $\mu_{R}$, and factorisation scale, $\mu_{F}$.

The correct description of the vector-boson transverse momentum, $p_{T}(V=W, Z)$, is essential for the estimation of $A_{W}$ and $A_{Z}$. Since fixed order perturbative QCD predictions do not provide a sufficiently good description of the low $p_{T}(V=W, Z)$ spectrum, we use the POWHEG MC generator interfaced to PYTHIA8, henceforth referred to as POWHEG+PYTHIA8, to estimate the central values for $A_{W}$ and $A_{Z}$, using the MMHT2014 PDF set.

The uncertainties due to missing higher order QCD corrections are estimated by varying the renormalisation and factorisation scales, $\mu_{R}$ and $\mu_{F}$, by a factor of two up and down, as well as by reevaluating the acceptance factors with hdamp set to $m_{V}(V=W, Z)$, instead of the default value hdamp $=\infty$ [25], in the PowHEG generator. The correlation of the $\mu_{R}$ and $\mu_{F}$ variations on the $W$ - and $Z$-boson cross sections and acceptances can be treated according to various prescriptions. In the most conservative approach they are considered as fully uncorrelated, leading to an uncertainty of $0.5 \%$ on the predicted inclusive cross-section ratio. The uncertainty reduces by more than a factor of two when assuming a fully correlated behaviour. In the following we adopt an intermediate approach, and assume a correlation of $50 \%$. In addition to these uncertainties, the acceptance factor ratio $A_{W} / A_{Z}$ is also affected by other effects, which change the kinematic distribution of the final states, but has little effect on the inclusive cross sections. In particular, the uncertainties due to soft non-perturbative effects and initial-state radiation (ISR), which vary the transverse momentum spectrum of the vector boson, $p_{T}(V)$, have to be estimated. To perform a conservative estimation, we reweight the predicted $p_{T}(V)$ from POWHEG+PYTHIA8 to corresponding predictions of the RESBOS generator [26-28]. RESBOS is based on a resummed calculation, which is performed at next-to-next-to-leading logarithmic order and matched to approximate NNLO perturbative QCD calculations at large boson momenta. The difference between the nominal POWHEG+PYTHIA8 predictions of $A_{W}$ and $A_{Z}$ and the RESBOS reweighted samples, is considered as an ISR and resummation uncertainty. The corresponding uncertainties on $A_{W} / A_{Z}$ vary between $0.1 \%$ (ATLAS) and $0.4 \%$ (CMS). This difference can be explained by the larger effect on the $W$-boson selection for CMS, as it

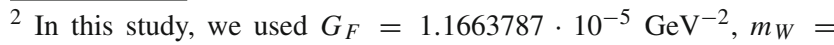
$80385 \mathrm{MeV}, m_{Z}=91187.6 \mathrm{MeV}$, and $\Gamma_{Z}=2495 \mathrm{MeV}$.
} 
Table 1 The collider beams, the corresponding centre-of-mass energy, the fiducial volume definitions, the published inclusive cross-section ratio $R$, as well as the fiducial cross-section ratio $R_{\text {fid }}$ are given for each analysis used for the combination. The fiducial ratio was not published for the measurements of the $\mathrm{CDF}$ experiment and the extrapolated value is shown

\begin{tabular}{|c|c|c|c|c|}
\hline Experiment & Collider & Fiducial volume definition & $R$ (published) & $R_{\text {fid }}$ \\
\hline CDF [14] & $p \bar{p}, \sqrt{s}=1.96 \mathrm{TeV}$ & $\begin{array}{l}p_{T}^{\mu}>20 \mathrm{GeV} \text { for }|\eta|<1.0 \\
Z: 66<m_{e e}<116 \mathrm{GeV} \\
\mathrm{W}: p_{T}^{v}>20 \mathrm{GeV}\end{array}$ & $\begin{array}{l}10.93 \\
\pm 0.27 \text { (stat) } \\
\pm 0.18 \text { (sys) }\end{array}$ & $\begin{array}{l}7.46 \\
\pm 0.18 \text { (stat) } \\
\pm 0.12 \text { (sys) (extrapolated) }\end{array}$ \\
\hline ATLAS [21] & $p p, \sqrt{s}=7 \mathrm{TeV}$ & $\begin{array}{l}p_{T}^{\ell}>20 \mathrm{GeV} \\
\left|\eta_{\ell}\right|<2.5 \\
Z: 66<m_{l l}<116 \mathrm{GeV} \\
\mathrm{W}: p_{T}^{\nu}>25 \mathrm{GeV}, m_{T}>40 \mathrm{GeV}\end{array}$ & $\begin{array}{l}10.91 \\
\pm 0.11 \text { (stat) } \\
\pm 0.17 \text { (sys) }\end{array}$ & $\begin{array}{l}10.85 \\
\pm 0.11 \text { (stat) } \\
\pm 0.17 \text { (sys) (published) }\end{array}$ \\
\hline CMS [16] & $p p, \sqrt{s}=7 \mathrm{TeV}$ & $\begin{array}{l}Z: p_{T}^{\mu}>20 \mathrm{GeV} \text { for }|\eta|<2.1 \\
Z: 60<m_{e e}<120 \mathrm{GeV} \\
\mathrm{W}: p_{T}^{\mu}>25 \mathrm{GeV} \text { for }|\eta|<2.1\end{array}$ & $\begin{array}{l}10.52 \\
\pm 0.09 \text { (stat) } \\
\pm 0.10 \text { (sys) }\end{array}$ & $\begin{array}{l}11.95 \\
\pm 0.10 \text { (stat) } \\
\pm 0.20 \text { (sys) (published) }\end{array}$ \\
\hline CMS [17] & $p p, \sqrt{s}=8 \mathrm{TeV}$ & $\begin{array}{l}Z: p_{T}^{\mu}>25 \mathrm{GeV} \text { for }|\eta|<2.1 \\
Z: 60<m_{e e}<120 \mathrm{GeV} \\
\mathrm{W}: p_{T}^{\mu}>25 \mathrm{GeV} \text { for }|\eta|<2.1\end{array}$ & $\begin{array}{l}10.44 \\
\pm 0.14 \text { (stat) } \\
\pm 0.30 \text { (sys) }\end{array}$ & $\begin{array}{l}13.28 \\
\pm 0.18 \text { (stat) } \\
\pm 0.23 \text { (sys) (published) }\end{array}$ \\
\hline
\end{tabular}

Table 2 Predicted acceptance ratios $A_{W} / A_{Z}$ for the extrapolation from the experimental fiducial region to the full phase space and predicted cross-section ratios $\sigma_{W} / \sigma_{Z}$ for all measurements under consideration. In addition, the uncertainties due to initial-state radiation modelling

\begin{tabular}{llllllrl}
\hline Experiment & Quantity & Value & Scales $\left(\mu_{R}, \mu_{F}\right)$ & ISR+ resummation & PDF & QED FSR + EWK & Total \\
\hline CDF & $A_{W} / A_{Z}$ & 1.884 & 0.007 & 0.003 & 0.006 & 0.004 & 0.011 \\
& $\left(\sigma_{W} / \sigma_{Z}\right)_{\text {pred }}$ & 3.391 & 0.005 & - & 0.013 & - & 0.014 \\
ATLAS & $A_{W} / A_{Z}$ & 1.000 & 0.003 & 0.002 & 0.004 & 0.002 & - \\
& $\left(\sigma_{W} / \sigma_{Z}\right)_{\text {pred }}$ & 3.395 & 0.012 & - & 0.020 & - \\
CMS $(7 \mathrm{TeV})$ & $A_{W} / A_{Z}$ & 1.135 & 0.003 & 0.006 & 0.005 & 0.002 & -0.023 \\
& $\left(\sigma_{W} / \sigma_{Z}\right)_{\text {pred }}$ & 3.346 & 0.012 & - & 0.019 & - & 0.008 \\
CMS $(8 \mathrm{TeV})$ & $A_{W} / A_{Z}$ & 1.266 & 0.004 & 0.007 & 0.005 & 0.002 & 0.022 \\
& $\left(\sigma_{W} / \sigma_{Z}\right)_{\text {pred }}$ & 3.326 & 0.013 & - & 0.020 & - & 0.009 \\
& & & & & 0.023 \\
\hline
\end{tabular}

and resummation model (ISR), factorisation and renormalisation scales, PDF, QED final state radiation uncertainties as well as electroweak corrections are given. It should be noted that the invariant mass requirement of the $Z$-boson selection is different between the analyses requires only a minimum threshold on the $p_{T}$ of the decay muons.

Furthermore, NLO electroweak corrections can be comparable in size to NNLO QCD corrections. We distinguish between the corrections due to QED final-state radiation (FSR) and loop-induced electroweak corrections (EWK). The QED FSR related uncertainties are estimated by comparing SHERPA [29] and PYTHIA8 [30], where the acceptances are derived for both generators using dressed and bare leptons. The resulting differences in the predicted acceptance ratios are taken as the QED FSR model uncertainty and amount to $0.1 \%$. The uncertainties due to loop-induced electroweak corrections are taken from literature [31] and are accounted for by $0.1 \%$ variations on $A_{W} / A_{Z}$.
We obtain 57 predictions for the cross-section ratios $\sigma_{W} / \sigma_{Z}$ and the acceptance ratios $A_{W} / A_{Z}$, accounting for 50 MMHT PDF eigenvector variations, the central prediction of the CT10 PDF set, $\mu_{R}$ and $\mu_{F}$ scale variations, and variations of $\alpha_{s}$. In addition, we have further uncertainties on $A_{W} / A_{Z}$ due to ISR/resummation effects, QED FSR and NLO EWK effects. A summary of the cross-section ratios $\sigma_{W} / \sigma_{Z}$ and acceptance ratios $A_{W} / A_{Z}$ for each measurement, including the relevant model uncertainties, is given in Table 2. The PDF uncertainties are evaluated with the Hessian method [32].

The uncertainties due to ISR and resummation, QED FSR, and electroweak corrections, as well as the variations of $\mu_{F}$ and $\mu_{R}$, are symmetrised by taking the average of the positive and negative variations. 
Table 3 Extracted values of $\mathrm{BR}(W \rightarrow \mu \nu)(\%)$ for all four measurements and their combination. The associated statistical, experimental, and model uncertainties are also given

\begin{tabular}{|c|c|c|c|c|c|c|c|c|}
\hline Experiment & BR. (\%) & Stat. & Exp. sys. & Scales $\left(\mu_{R}, \mu_{F}\right)$ & ISR+ resummation & PDF & FSR + EWK & Total \\
\hline ATLAS & 10.75 & 0.11 & 0.17 & 0.05 & 0.02 & 0.10 & 0.01 & 0.23 \\
\hline CMS (7 TeV) & 10.59 & 0.09 & 0.18 & 0.05 & 0.05 & 0.10 & 0.02 & 0.23 \\
\hline CMS $(8 \mathrm{TeV})$ & 10.69 & 0.14 & 0.19 & 0.06 & 0.05 & 0.11 & 0.02 & 0.27 \\
\hline $\mathrm{CDF}$ & 11.06 & 0.27 & 0.18 & 0.04 & 0.02 & 0.07 & 0.02 & 0.33 \\
\hline Combined & 10.72 & 0.07 & 0.09 & 0.04 & 0.02 & 0.10 & 0.02 & 0.16 \\
\hline
\end{tabular}

\section{Extraction of the $W$-boson width and combination}

The total inclusive cross-section ratio for each experiment is estimated by combining the central values of $A_{W} / A_{Z}$ reported in Table 2 and the fiducial cross-section ratios $R_{\mathrm{fid}}$ from Table 1. It should be noted that these derived values for $R$ will differ from the original published values, as our baseline prediction for the estimation of $A_{W} / A_{Z}$ differs from the approach followed by each experiment. Clearly, the advantage of having a common model for the theoretical predictions lies in the traceability of correlated systematic uncertainties. The published values of $R$ are compared to the values obtained using the newly derived acceptance ratios as a first sanity check of our extrapolation. The derived values agree with the published values of the experiments within their associated model uncertainties.

In a second step, the expected leptonic branching ratios can be rederived for each experiment individually, using the predicted cross-section ratios, the measured fiducial ratios of the experiments reported in Table 2, and the relation

$\mathrm{BR}(W \rightarrow \mu \nu)=\frac{\Gamma_{W \rightarrow \mu \nu}}{\Gamma_{W}}=R \cdot \frac{\sigma_{Z}}{\sigma_{W}} \cdot \frac{\Gamma_{Z \rightarrow \mu \mu}}{\Gamma_{Z}}$,

where a value of the leptonic $Z$-boson branching ratio of $\Gamma_{Z \rightarrow \mu \mu} / \Gamma_{Z}=0.033658 \pm 0.000023$ [12] is used. The results are presented in Table 3. Assuming the validity of the SM, the partial leptonic $W$-boson width is predicted by Eq. (1), leading to $\Gamma_{W \rightarrow \mu \nu}=226.5 \mathrm{MeV}$, where the $\left(1+\delta^{\mathrm{rad}}\right)$ corrections are taken from Ref. [2]. Finally, the total $W$-boson width can be derived from the measured leptonic branching ratios. The resulting values for $\Gamma_{W}=\mathrm{BR} \cdot \Gamma_{W \rightarrow \mu \nu}$ for each experimental measurement are illustrated in Fig. 2 and reported in Table 4, together with the associated statistical, experimental systematic, and combined model uncertainties.

For the combination, we use the measured inclusive crosssection ratio $R$ and the corresponding inclusive cross-section prediction for each model systematic variation, thus leading to new values of $\mathrm{BR}(W \rightarrow \mu \nu)$ and $\Gamma_{W}$ for each measurement, respectively. In a second step, we combine the individual measurements following the BLUE method [33], again, separately for all model variations. For the combination of the four experimental values of $\mathrm{BR}(W \rightarrow \mu \nu)$ and $\Gamma_{W}$, we treat the statistical and experimental systematic uncertainties as fully uncorrelated. In a last step, we calculate the difference between the combined values of $\mathrm{BR}(W \rightarrow \ell v)$ and $\Gamma_{W}$ for each model variation and their central combined values, and evaluate the theoretical and model systematic uncertainties from these differences. The PDF uncertainties are evaluated with the MMHT2014 PDF set using the Hessian method [32]. The other model systematic uncertainties are added in quadrature.

The results of the combination are

$$
\begin{aligned}
\mathrm{BR}(W \rightarrow \mu \nu) & =(10.72 \pm 0.07 \pm 0.09 \pm 0.11) \% \\
& =(10.72 \pm 0.16) \%
\end{aligned}
$$

and

$$
\begin{aligned}
\Gamma_{W} & =2113 \pm 13 \pm 18 \pm 22 \mathrm{MeV} \\
& =2113 \pm 31 \mathrm{MeV},
\end{aligned}
$$

where the first uncertainty is statistical, the second is the experimental systematic uncertainty, and the third is the modelling systematic uncertainty. The results are shown and compared to the current world averages and to the SM predictions in Figs. 1 and 2, respectively. The statistical and systematic uncertainties of the combined measurement of $\Gamma_{W}$ are $30 \%$ and $45 \%$ smaller, respectively, compared to the uncertainties of the most precise single measurement. The model uncertainties on the combined values do not significantly change and are dominated by PDF uncertainties.

\section{Summary and interpretation}

In this paper we have presented a combination of measurements of the muonic branching ratio of the $W$ boson and its total decay width, extracted from the cross-section ratios of $W$ - and $Z$-boson production from the ATLAS, CMS, and $\mathrm{CDF}$ experiments at various centre-of-mass energies. Special emphasis was drawn to the correct treatment of the correlations between systematic uncertainties, in particular uncertainties due to the limited knowledge of the parton distribution functions and variations of the renormalisation 
Table 4 Extracted values of $\Gamma_{W}(\mathrm{MeV})$ for all four measurements and their combination. The associated statistical, experimental, and model uncertainties are also given

\begin{tabular}{|c|c|c|c|c|c|c|c|c|}
\hline Experiment & $\Gamma_{W}(\mathrm{MeV})$ & Stat. & Exp. sys. & Scales $\left(\mu_{R}, \mu_{F}\right)$ & ISR + resummation & PDF & FSR + EWK & Total \\
\hline ATLAS & 2108 & 21 & 33 & 10 & 3 & 18 & 4 & 44 \\
\hline CMS (7 TeV) & 2140 & 18 & 36 & 9 & 11 & 18 & 4 & 46 \\
\hline CMS (8 TeV) & 2120 & 29 & 37 & 12 & 11 & 19 & 4 & 53 \\
\hline $\mathrm{CDF}$ & 2050 & 51 & 34 & 8 & 4 & 13 & 4 & 63 \\
\hline Combined & 2113 & 13 & 18 & 8 & 6 & 19 & 4 & 31 \\
\hline
\end{tabular}

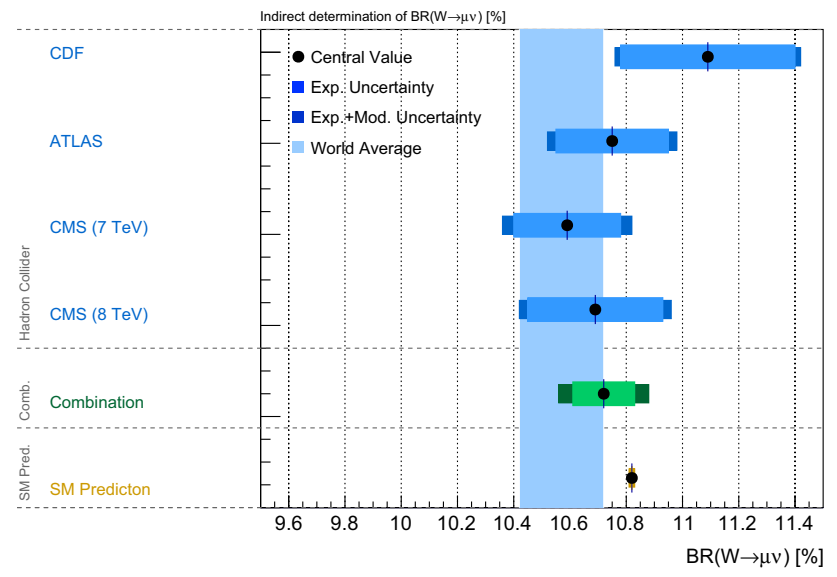

Fig. 1 Reestimated $\mathrm{BR}(W \rightarrow \mu \nu)$ values of the measurements under consideration as well as the combined value, the SM prediction, and the current world average

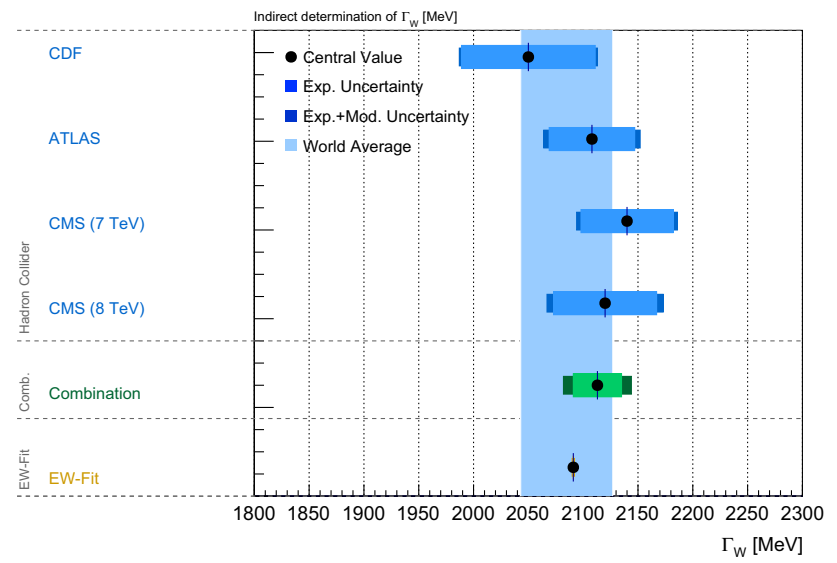

Fig. 2 Reestimated $W$-boson width values of the measurements under consideration as well as the combined value, the result of the global electroweak fit, and the current world average

and factorisation scales. The combination yields $\mathrm{BR}(W \rightarrow$ $\mu \nu)^{R}=(10.72 \pm 0.16) \%$ and $\Gamma_{W}^{R}=2113 \pm 31 \mathrm{MeV}$. The results are compatible in value, and similar in accuracy, to the current world averages $\mathrm{BR}(W \rightarrow \mu \nu)^{\mathrm{WA}}=(10.57 \pm$ $0.15) \%[11,34-37]$ and $\Gamma_{W}^{\mathrm{WA}}=2085 \pm 42 \mathrm{MeV}[6,11]$, which are based solely on direct measurements.
The total width of the $W$ boson is potentially sensitive to new physics scenarios in the context of the global electroweak fit [38]. The indirect determination via the electroweak fit yields a value of $\Gamma_{W}^{E W}=2091 \pm 1 \mathrm{MeV}$ [39], which is in good agreement with our combined value. The relation expressed in Eq. (1) shows that $\Gamma_{W}$ depends, among other SM parameters, on $m_{W}, \alpha_{s}$, and $m_{H}$. However, the small uncertainties on the determination of $\Gamma_{W}^{E W}$ indicates that the sensitivity of $\Gamma_{W}$ to these parameters of the SM is rather weak.

It should be noted that loop corrections arising from contributions of new physics to the $W$-boson width would alter the term $\delta^{\text {rad }}$ in Eq. (1) independently from the decay channel. As a consequence, the branching ratio is insensitive to effects that could appear in the corresponding loop correction terms. Only new physics effects that directly alter the leptonic branching ratio can be tested with our combined value $\mathrm{BR}(W \rightarrow \mu \nu)^{R}$. We find a perfect agreement with the $\mathrm{SM}$ prediction $\mathrm{BR}(W \rightarrow \mu \nu)^{S M}=(10.83 \pm 0.01) \%[1,2] .^{3}$

Acknowledgements We would like to thank M. Boonekamp, A. Glazov, K. Moenig, and S. Webb for the useful comments during the preparation this paper. This work was partly supported by the Volkswagen Foundation and the German Research Foundation (DFG).

Open Access This article is distributed under the terms of the Creative Commons Attribution 4.0 International License (http://creativecomm ons.org/licenses/by/4.0/), which permits unrestricted use, distribution, and reproduction in any medium, provided you give appropriate credit to the original author(s) and the source, provide a link to the Creative Commons license, and indicate if changes were made.

Funded by SCOAP ${ }^{3}$.

\section{References}

1. J.L. Rosner, M.P. Worah, T. Takeuchi, Oblique corrections to the $W$ width. Phys. Rev. D 49, 1363-1369 (1994). arXiv:hep-ph/9309307

2. P. Renton, Updated SM calculations of $\sigma_{W}$ boson width. arXiv:0804.4779 [hep-ph]

3. D. d'Enterria, M. Srebre, $\alpha_{s}$, and CKM unitarity test from $W$ decays at NNLO. arXiv:1603.06501 [hep-ph]

\footnotetext{
$\overline{{ }^{3} \text { Using } \alpha_{s}\left(m_{W}\right)}=0.1203$, corresponding to the value of $\alpha_{S}\left(m_{Z}\right)$ used for the MMHT2014 PDF set, the SM prediction of $\mathrm{BR}(W \rightarrow \mu \nu)^{S M}$ changes to $10.82 \%$.
} 
4. CDF Collaboration, T. Aaltonen et al., A direct measurement of the $W$ boson width in $p \bar{p}$ collisions at $\sqrt{s}=1.96-\mathrm{TeV}$. Phys. Rev. Lett. 100, 071801 (2008). arXiv:0710.4112 [hep-ex]

5. D0 Collaboration, V. M. Abazov et al., Direct measurement of the $W$ boson width. Phys. Rev. Lett. 103, 231802 (2009). arXiv:0909.4814 [hep-ex]

6. T. E. W. Group, Combination of CDF and D0 Results on the Width of the $W$ boson. arXiv:1003.2826 [hep-ex]

7. ALEPH Collaboration, S. Schael et al., Measurement of the $W$ collisions at LEP. Eur. Phys. J. C 47, 309-335 (2006). arXiv:hep-ex/0605011

8. DELPHI Collaboration, J. Abdallah et al., Measurement of the mass and width of the $W=161-\mathrm{GeV}-209-\mathrm{GeV}$. Eur. Phys. J. C 55, 1-38 (2008). arXiv:0803.2534 [hep-ex]

9. L3 Collaboration, P. Achard et al., Measurement of the mass and the width of the $W$ boson at LEP. Eur. Phys. J. C 45, 569-587 (2006). arXiv:hep-ex/0511049

10. OPAL Collaboration, G. Abbiendi et al., "Measurement of the mass and width of the $W$ boson," Eur. Phys. J. C45 (2006) 307-335, arXiv:hep-ex/0508060 [hep-ex]

11. DELPHI, OPAL, LEP Electroweak, ALEPH, L3 Collaboration, S. Schael et al., Electroweak measurements in electron-positron collisions at $W$-boson-pair energies at LEP. Phys. Rep. 532, 119244 (2013). arXiv:1302.3415 [hep-ex]

12. Particle Data Group Collaboration, K. Olive et al., Review of particle physics. Chin. Phys. C 38, 090001 (2014)

13. S. Catani, L. Cieri, G. Ferrera, D. de Florian, M. Grazzini, Vector boson production at hadron colliders: a fully exclusive QCD calculation at NNLO. Phys. Rev. Lett. 103, 082001 (2009). arXiv:0903.2120 [hep-ph]

14. CDF Collaboration, A. Abulencia et al., "Measurements of inclusive $\mathrm{W}$ and $\mathrm{Z}$ cross sections in $\mathrm{p}$ anti-p collisions at $\sqrt{s}=$ 1.96 TeV," J. Phys. G 34 (2007) 2457-2544, arXiv:hep-ex/0508029 [hep-ex]

15. D0 Collaboration, B. Abbott et al., Extraction of the width of the $W$ and their ratio. Phys. Rev. D 61, 072001 (2000). arXiv:hep-ex/9906025

16. CMS Collaboration, S. Chatrchyan et al., Measurement of the inclusive $W$ and $Z$ production cross sections in $p p$ collisions at $\sqrt{s}=7$ TeV. JHEP 10, 132 (2011). arXiv:1107.4789 [hep-ex]

17. C.M.S. Collaboration, S. Chatrchyan et al., Measurement of inclusive $W$ and $Z$ boson production cross sections in pp collisions at $\sqrt{s}=8$ TeV. Phys. Rev. Lett. 112, 191802 (2014). arXiv:1402.0923 [hep-ex]

18. S.D. Drell, T.-M. Yan, Massive lepton pair production in hadronhadron collisions at high-energies. Phys. Rev. Lett. 25, 316-320 (1970) (erratum: Phys. Rev. Lett. 25, 902, 1970)

19. T. Sjostrand, L. Lonnblad, S. Mrenna, PYTHIA 6.2: physics and manual. arXiv:hep-ph/0108264

20. CTEQ Collaboration, H. Lai et al., Global QCD analysis of parton structure of the nucleon: CTEQ5 parton distributions. Eur. Phys. J. C 12, 375-392 (2000). arXiv:hep-ph/9903282

21. ATLAS Collaboration, G. Aad et al., Measurement of the inclusive $W^{ \pm} \mathrm{TeV}$ with the ATLAS detector. Phys. Rev. D 85, 072004 (2012). arXiv:1109.5141 [hep-ex]

22. R. Gavin, Y. Li, F. Petriello, S. Quackenbush, FEWZ 2.0: a code for hadronic $Z$ production at next-to-next-to-leading order. Comput. Phys. Commun. 182, 2388-2403 (2011). arXiv:1011.3540 [hep$\mathrm{ph}]$
23. L.A. Harland-Lang, A.D. Martin, P. Motylinski, R.S. Thorne, Parton distributions in the LHC era: MMHT 2014 PDFs. Eur. Phys. J. C 75(5), 204 (2015). arXiv:1412.3989 [hep-ph]

24. J. Gao et al., CT10 next-to-next-to-leading order global analysis of QCD. Phys. Rev. D 89(3), 033009 (2014). arXiv:1302.6246 [hep$\mathrm{ph}]$

25. S. Alioli, P. Nason, C. Oleari, E. Re, NLO Higgs boson production via gluon fusion matched with shower in POWHEG. JHEP 04, 002 (2009). arXiv:0812.0578 [hep-ph]

26. C. Balazs, C.P. Yuan, Soft gluon effects on lepton pairs at hadron colliders. Phys. Rev. D 56, 5558-5583 (1997). arXiv:hep-ph/9704258

27. G.A. Ladinsky, C.P. Yuan, The nonperturbative regime in QCD resummation for gauge boson production at hadron colliders. Phys. Rev. D 50, 4239 (1994). arXiv:hep-ph/9311341

28. M. Guzzi, P.M. Nadolsky, B. Wang, Nonperturbative contributions to a resummed leptonic angular distribution in inclusive neutral vector boson production. Phys. Rev. D 90(1), 014030 (2014). arXiv:1309.1393 [hep-ph]

29. T. Gleisberg, S. Hoeche, F. Krauss, M. Schonherr, S. Schumann, F. Siegert, J. Winter, Event generation with SHERPA 1.1. JHEP 02, 007 (2009). arXiv:0811.4622 [hep-ph]

30. T. Sjostrand, S. Mrenna, P.Z. Skands, A brief introduction to PYTHIA 8.1. Comput. Phys. Commun. 178, 852-867 (2008). arXiv:0710.3820 [hep-ph]

31. G. Balossini, G. Montagna, C.M. Carloni Calame, M. Moretti, M. Treccani, O. Nicrosini, F. Piccinini, A. Vicini, Electroweak \& QCD corrections to Drell Yan processes. Acta Phys. Polon. B 39, 1675 (2008). arXiv:0805.1129 [hep-ph]

32. J. Pumplin, D. Stump, R. Brock, D. Casey, J. Huston, J. Kalk, H.L. Lai, W.K. Tung, Uncertainties of predictions from parton distribution functions. 2. The Hessian method. Phys. Rev. D 65, 014013 (2001). arXiv:hep-ph/0101032

33. L. Lyons, D. Gibaut, P. Clifford, How to combine correlated estimates of a single physical quantity. Nucl. Instrum. Methods A 270 , 110 (1988)

34. ALEPH Collaboration, A. Heister et al., Measurement of $W$ collisions at centre-of-mass energies from $183-\mathrm{GeV}$ to $209-\mathrm{GeV}$. Eur. Phys. J. C 38, 147-160 (2004)

35. DELPHI Collaboration, J. Abdallah et al., Measurement of the $W$ pair production cross-section and $W$ branching ratios in e+ $\mathrm{e}-$ collisions at $s * *(1 / 2)=161-\mathrm{GeV}$ to $209-\mathrm{GeV}$. Eur. Phys. J. C 34, 127-144 (2004). arXiv:hep-ex/0403042

36. L3 Collaboration, P. Achard et al., Measurement of the cross section of $W$-boson pair production at LEP. Phys. Lett. B 600, 22-40 (2004). arXiv:hep-ex/0409016

37. OPAL Collaboration, G. Abbiendi et al., Measurement of the e+ decay branching fractions at LEP. Eur. Phys. J. C 52, 767-785 (2007). arXiv:0708.1311 [hep-ex]

38. ALEPH, CDF, D0, DELPHI, L3, OPAL, SLD, LEP Electroweak Working Group, Tevatron Electroweak Working Group, SLD Electroweak and Heavy Flavour Groups Collaboration, L. E. W. Group, Precision electroweak measurements and constraints on the standard model. arXiv:1012.2367 [hep-ex]

39. Gfitter Group Collaboration, M. Baak et al., The global electroweak fit at NNLO and prospects for the LHC and ILC. Eur. Phys. J. C 74, 3046 (2014). arXiv:1407.3792 [hep-ph] 